\title{
Bilateral Bizarre Vulvar Swellings in a Teenager: A very rare Entity of Aggressive Angiomyxoma
}

\author{
Tashhir Rana* and Abeer Yasin
}

Radiology Department, Sharif Medical City Hospital, Lahore, Pakistan

*Corresponding author: Tashhir Rana, Professor and Head of Radiology Department, Sharif Medical City Hospital, Lahore, Pakistan, E-mail: tashhir.rabbani@gmail.com

\begin{abstract}
Background: Aggressive angiomyxoma (AA) is a rare mesenchymal neoplasm that commonly occurs in perineal region of females.

Case Report: A 17 years female presents with bilateral bizarre vulvar swellings, gradually increasing in size for 2 years, painful for 1 week. Sonographic evaluation revealed bilateral well-defined hypoechoic lesions with internal vascularity and cystic areas. Magnetic resonance imaging (MRI) demonstrated these lesions to be iso-intense to skeletal muscles on T1-weighted images (T1WI) and hyperintense on T2-weighted images (T2WI). These were surgically excised and sent for histopathology that confirmed these lesions to be AA.

Discussion: AA is an uncommon neoplasm of mesenchymal origin that arises mainly in the perineal soft tissue of adults. It occurs predominantly in females with a peak incidence in fourth decade. [1] In our case the patient was a teenager. In females it may present as a vulvar nodule, polyp or may even be mistaken for a Bartholin's or labial cyst. In males it involves analogous sites including inguino-scrotal region. [4] Most of the reported cases include solitary unilateral lesions.
\end{abstract}

Conclusion: Our case is unique because the patient was in her teenage and presented with bilateral vulvar angiomyxomas that showed internal vessels on colour doppler imaging.

Keywords: Case report; Aggressive angiomyxoma; Vulvar swelling; Teenager; Sonography, CT, MRI

Received Date: October 11, 2019; Accepted Date: October 25, 2019; Published Date: November 01, 2019

\section{Abbreviations}

AA: Aggressive Angiomyxoma; MRI: Magnetic Resonance Imaging; T1WI: T1-weighted Images; T2WI: T2-weighted Images; T2WI/FAT-SAT: T2-weighted Fat Suppressed Images; AP: Anteroposterior; CC: Craniocaudal; CT: Computed Tomography

Citation: Tashhir Rana, Bilateral Bizarre Vulvar Swellings in a Teenager: A very rare Entity of Aggressive Angiomyxoma. J Clin Cases Rep 3(3): 82-88. 


\section{Introduction}

Aggressive angiomyxoma is a rare mesenchymal neoplasm that arises in the soft tissues. It commonly occurs in the perineal region of females [1]. Steeper and Rosai were the first ones to describe this benign myxoid and vascular tumor in a case series of nine patients in 1983 [2]. The term aggressive demonstrates the infiltrative nature of the neoplasm that has propensity of local recurrence [3]. Sutton et al. stated that less than 350 cases have been reported in literature till 2012 with female to male ratio of $6.6: 1[1]$.

\section{Case Report}

A 17 years old female patient was referred from Gynaecology department for abdominopelvic ultrasound in January, 2016 with complaint of bilateral vulvar swellings gradually increasing in size for 2 years, more on right side that was painful for 1 week. The patient had menarche at 13 years of age with a normal 30/6 days menstrual cycle.

On examination there was swelling of right labium majus with a polypoidal outgrowth from its inferolateral margin measuring about $9 \mathrm{~cm}$ in length and $2 \mathrm{~cm}$ in diameter. It was spongy in consistency with no cutaneous darkening, papules or ulceration. Left labium majus was also swollen with a $2 \mathrm{~cm} \times 1.5 \mathrm{~cm}$ palpable lesion having similar characteristics. These lesions had striking resemblance with male external genitalia, the labial swellings appearing as rudimentary scrotal sacs and the polypoidal outgrowth conforming to the shape of rudimentary glans and penile shaft. However the vagina and urethra were unremarkable (Figure 1).

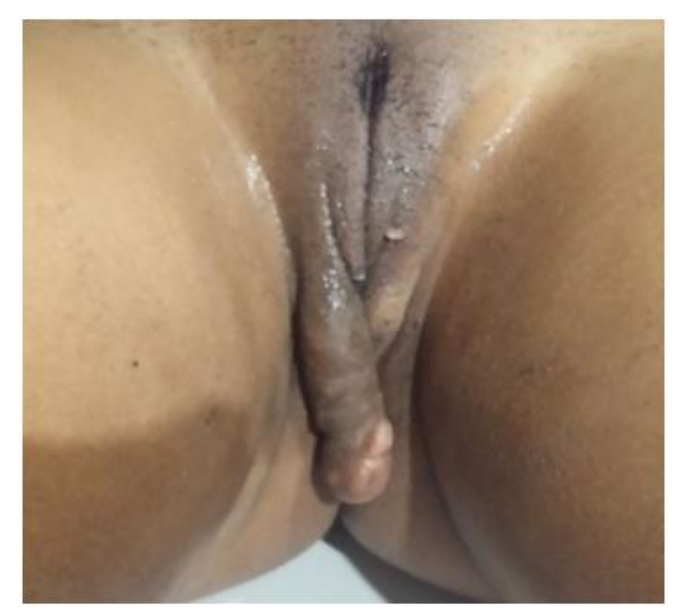

Figure 1: Showing bilateral labial swellings with a polypoidal outgrowth from inferolateral margin of right labium majus.

\section{Lab investigations}

Lab investigations were normal. On ultrasound the right labial swelling consisted of a well-defined hypoechoic lesion with internal vascularity. The polypoidal outgrowth consisted of a well-defined hypoechoic lesion with internal septated cystic areas and was relatively avascular. Left labial swelling also consisted of a well-defined hypoechoic lesion with blood flow on colour doppler. Uterus was anteverted, normal sized with central endometrium. No endomyometrial pathology was detected. Both ovaries were normally visualized with growing follicles in each. Abdominal scan had nothing to add (Figure 2 - Figure 6). 


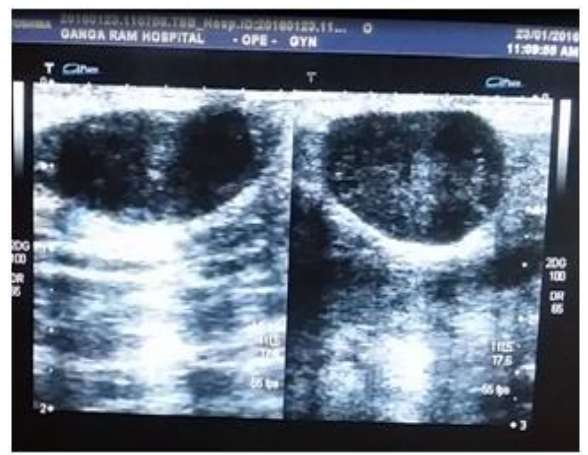

Figure 2: Grey scale ultrasound images of the right and left vulvar swelling respectively demonstrate well-defined ovoid hypoechoic lesions in the subcutaneous plane.

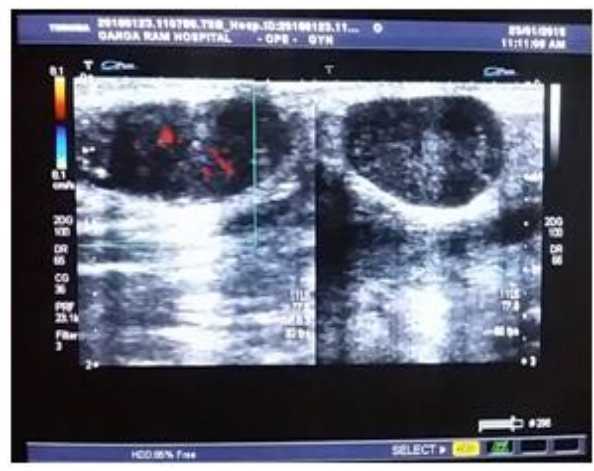

Figure 3: Colour Doppler imaging of the right vulvar swelling displays blood flow with vessels traversing through the lesion.
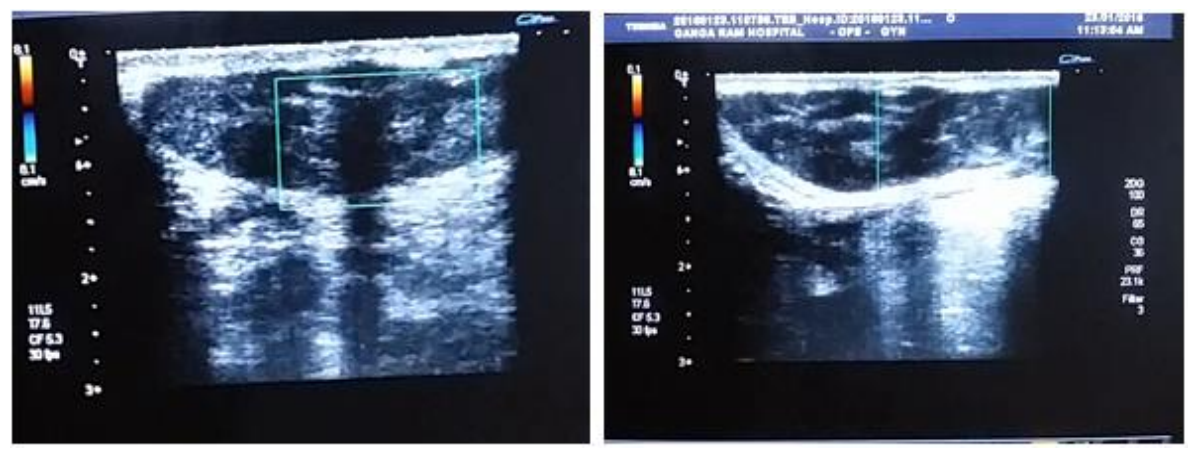

Figure 4: Colour Doppler imaging of the polypoidal outgrowth from right vulvar swelling demonstrates a well-defined hypoechoic lesion with internal anechoic cystic areas and septations displaying no vascularity.

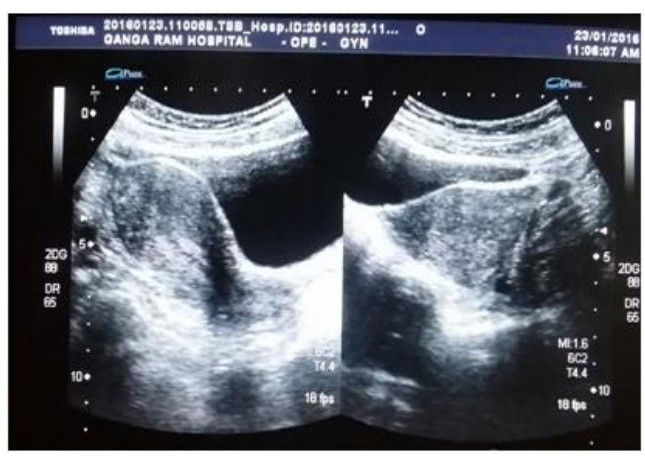

Figure 5: Grey scale ultrasound images of pelvis in longitudinal and horizontal planes respectively show normal-sized anteverted uterus with central endometrium. 


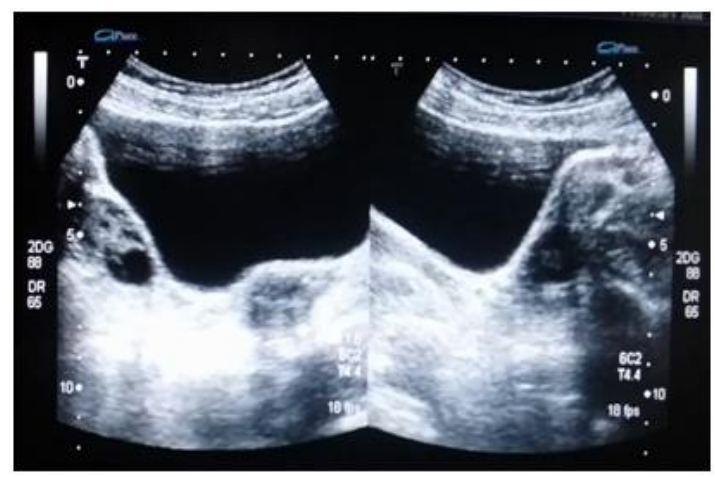

Figure 6: Grey scale ultrasound images of pelvis in horizontal plane show normal sized right and left ovaries respectively with growing follicles in each.

MRI showed a well-defined rounded lesion in right labium majus displaying iso-intense signals to skeletal muscles on T1WI and hyperintense signals on T2-weighted fat suppressed images (T2WI/FAT-SAT), measuring $10 \mathrm{~mm} \times 8 \mathrm{~mm}$ with a caudal extension consisting of multi-septated cystic lesion measuring $70 \mathrm{~mm} \times 21 \mathrm{~mm} \times 13 \mathrm{~mm}$ in anteroposterior (AP), craniocaudal (CC) and transverse dimensions respectively. The lesion in left labium majus is also well-defined and rounded with similar signal characters, measuring $19 \mathrm{~mm} \times 18 \mathrm{~mm} \times 9 \mathrm{~mm}$ in AP, CC and transverse dimensions respectively. No external or internal communication or extension noted (Figure 7a - Figure 7e).
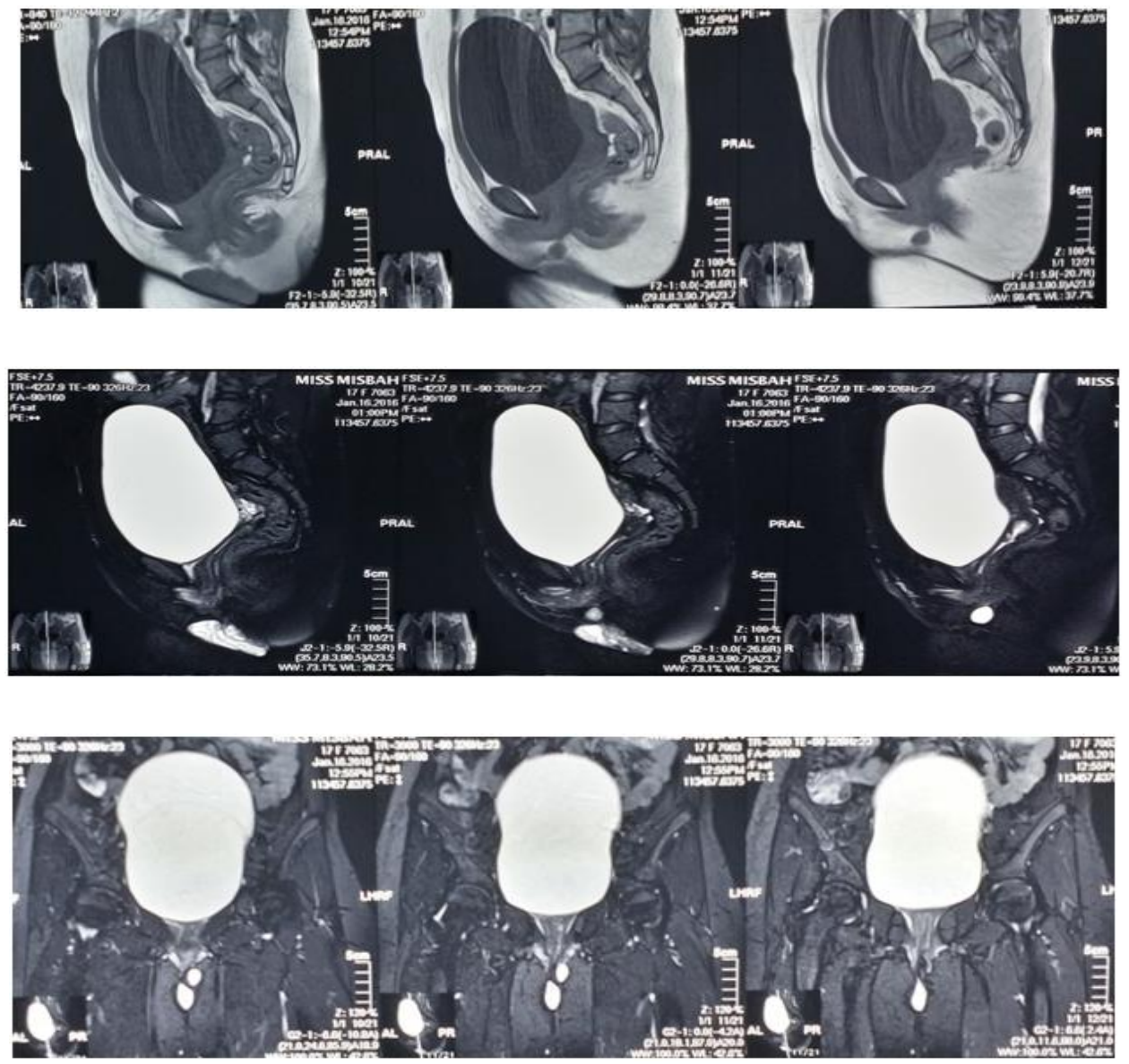

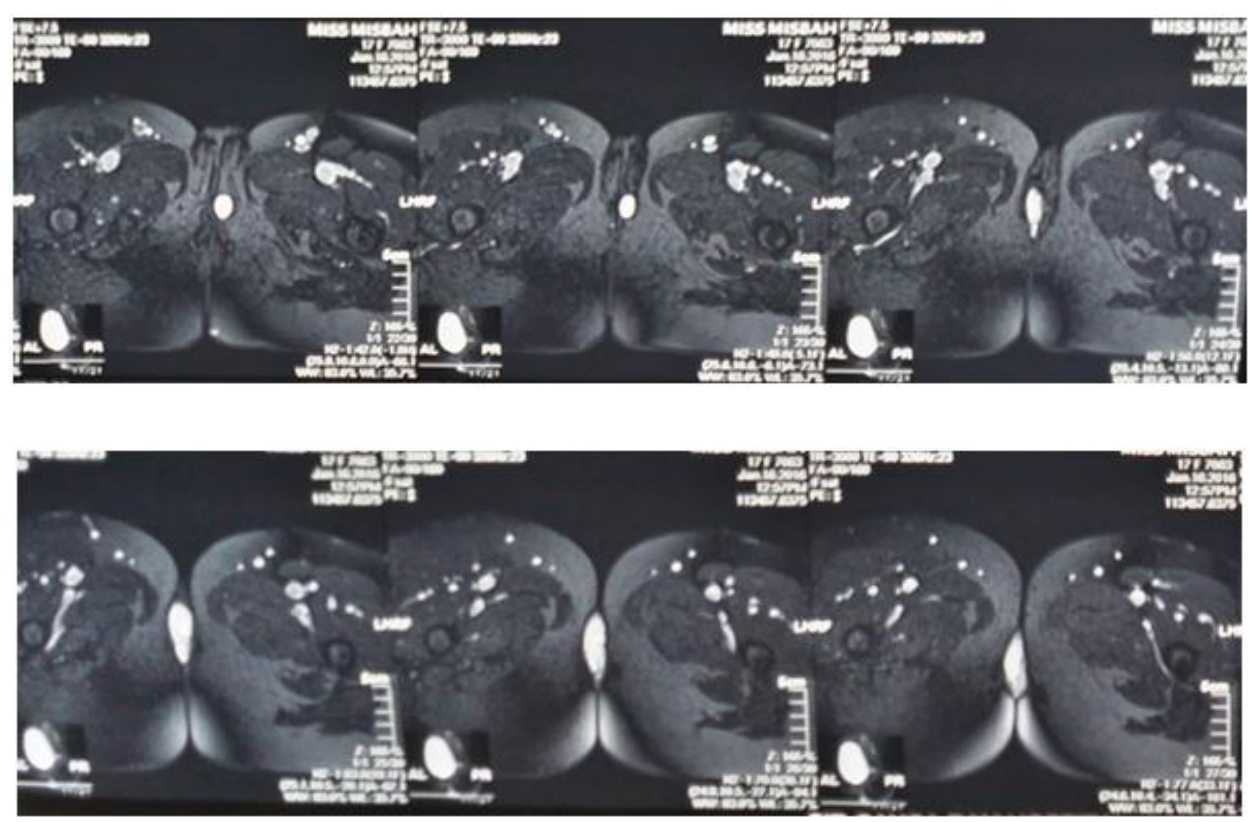

Figure 7A - Figure 7E: MR images of the lesion (A) Sagittal T1WI (B) Sagittal T2WI/FAT-SAT images (C) Coronal T2WI/FAT-SAT and (D,E) Axial T2WI/FAT-SAT.

Figure 7A-7E shows a well-defined rounded lesion in right labium majus displaying iso-intense signals to skeletal muscles on T1WI and hyperintense signals on T2WI/FAT-SAT, measuring $10 \mathrm{~mm} \times 8 \mathrm{~mm}$ with a caudal extension consisting of multiseptated cystic lesion measuring $70 \mathrm{~mm} \times 21 \mathrm{~mm} \times 13 \mathrm{~mm}$ in AP, CC and transverse dimensions respectively. The lesion in left labium majus is also well-defined and rounded with similar signal characters, measuring $19 \mathrm{~mm} \times 18 \mathrm{~mm} \times 9 \mathrm{~mm}$ in AP, $\mathrm{CC}$ and transverse dimensions respectively.

Based on these imaging findings the provisional diagnosis was of bilateral benign-appearing neoplastic lesions of soft tissue origin. However the patient was not ready for any invasive intervention and went to spiritual healers instead for pain relief. Two years later pain recurred and the patient presented again, now willing for surgical intervention. These lesions were then surgically excised with no post-operative complications. Histopathology demonstrated paucicellular mass with predominant myxoid matrix, internal thick-walled vascular channels and cystic areas. The diagnosis was aggressive angiomyxoma.

Follow up imaging was done on May, 2019 that showed no recurrence.

\section{Discussion}

Aggressive Angiomyxoma is an uncommon neoplasm of mesenchymal origin that arises mainly in the perineal soft tissue of adults. It occurs predominantly in females with a peak incidence in fourth decade [1]. In our case the patient was a teenager. In females it may present as a vulvar nodule, polyp or may even be mistaken for a Bartholin's or labial cyst. In males it involves analogous sites including inguino-scrotal region [4]. Most of the reported cases include solitary unilateral lesions, in our case it was two separate lesions involving bilateral labia.

Superficial angiomyxoma can occur as a part of Carney complex [5]. The term aggressive demonstrates the infiltrative nature of the neoplasm that has propensity of local recurrence [3]. 
http://www.tridhascholars.org | July-2020

Histologically angiomyxoma is described as a mesenchymal neoplasm with fibroblasts in a myxoid matrix. Vascular proliferation is common [6].

On sonography AA appears as a hypoechoic soft tissue mass that can have cystic components [4]. In our case the mass had cystic component and vascularity as well.

On computed tomography (CT) the appearances are variable, it may appear as a well-defined soft tissue mass, hypodense relative to skeletal muscles with mild post contrast enhancement or as a mixed density lesion with solid and cystic components $[4,7]$.

On MRI it is isointese to skeletal muscles on T1WI and hyperintense on T2WI with characteristic laminated appearance and intense post-contrast enhancement.

Further internal cystic degeneration may be present. Large peripheral feeding vessels or internal vessels may also be seen [7]. Surgery is the mainstay of treatment with wide local excision [3].

\section{Conclusion}

Most of the reported cases of aggressive angiomyxoma occurred in females in their third and fourth decade with unilateral involvement. Our case is unique because the patient was in her teenage and presented with bilateral vulvar angiomyxoma that showed internal vessels on colour doppler imaging.

\section{Consent for Publication}

Informed written consent was taken from the patient to publish this case and the picture in a medical journal.

\section{References}

1. Sutton BJ, Laudadio J (2012) Aggressive angiomyxoma. Archives of Pathology \& Laboratory Medicine 136(2): 217221.

2. Steeper TA, Rosai J (1983) Aggressive angiomyxoma of the female pelvis and perineum. Report of nine cases of a distinctive type of gynecologic soft-tissue neoplasm. The American Journal of Surgical Pathology 7(5): 463-475.

3. Benson JC, Gilles S, Sanghvi T, et al. (2016) Aggressive angiomyxoma: Case report and review of the literature. Radiology Case Reports 11(4): 332-335.

4. Haldar K, Martinek IE, Kehoe S (2010) Aggressive angiomyxoma: a case series and literature review. European Journal of Surgical Oncology 36(4): 335-339.

5. Zizi-Sermpetzoglou A, Myoteri D, Koulia K, et al. (2015) Aggressive angiomyxoma of the vulva: A bizarre perineal lesion. Case reports in Oncological Medicine 2015: 292304.

6. Narang S, Kohli S, Kumar V, et al. (2014) Aggressive angiomyxoma with perineal herniation. Journal of Clinical Imaging Science 4: 23.

7. Surabhi VR, Garg N, Frumovitz M, et al. (2014) Aggressive angiomyxomas: A comprehensive imaging review with clinical and histopathologic correlation. American Journal of Roentgenology 202(6): 1171-1178. 